\title{
Implementando metodologías de evaluación en proyectos de redes de saneamiento en el Máster Universitario en Ingeniería Hidráulica y Medio Ambiente. Caso de estudio.
}

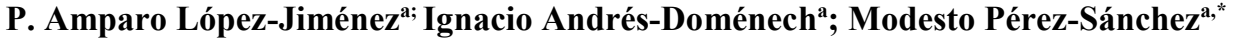 \\ a Departamento de Ingeniería Hidráulica y Medio Ambiente. Universitat Politècnica de València. \\ palopez@upv.es; igando@hma.upv.es; mopesan1@upv.es.
}

(*) autor de correspondencia

\begin{abstract}
Development of projects as teaching strategy is a powerful tool to activate and strengthen the students' learning. The classical evaluation of projects by numeric items has been the usual way but the introduction of UPV generic student outcomes within student's curricula obliges to update the evaluation criteria as well as the way to achieve it. This communication presents the time planning of a subject, which represents a level III (Master Degree) control point for the generic outcome 'Design and Project'. As a consequence of the wide range of the students background in this subject, there are many of them which did not previously work this outcome for levels I and II (Bachelor). A rubric was developed to do the evaluation in which descriptors were defined according to traditional used items. These defined descriptors were used to evaluate the reached degree by the students for each indicator. Finally, the experience demonstrated the possibility to evaluate the ability to develop a project using competences. A comparison between marks obtained by traditional system and marks obtained by competences was developed, getting similar results that did not change the final mark in the student curricula.
\end{abstract}

Keywords: hydraulic engineering, sewage network, descriptor, indicator

\footnotetext{
Resumen

El desarrollo de proyectos como estrategia de aprendizaje resulta una herramienta muy potente para activar y potenciar el aprendizaje de los alumnos. Tradicionalmente, la evaluación de proyectos mediante items numéricos ha sido la técnica más habitual pero la introducción de las competencias transversales en la evaluación del alumno, obliga a actualizar los criterios y formas de evaluación. La presente comunicación presenta la planificación temporal de una asignatura punto de control de Nivel de Dominio III (Master) con un gran número de alumnos que no han trabajo previamente los anteriores dominios. Dentro de la planificación, se encuentra la evaluación para la cual una rúbrica ha sido desarrollada, en la cual los descriptores contienen los items que tradicionalmente han sido
} 
evaluados y con los que ahora, se evalúa el grado alcanzado por el alumno en cada indicador. Finalmente, se demuestra que la evaluación del grado de adquisición de los resultados de aprendizaje a través de competencias es posible. Se realiza una comparación de notas obtenidas por el sistema tradicional y por competencias, obteniendo resultados muy similares, cuyas diferencias no afectan a la nota final del expediente del alumno.

Palabras clave: ingeniería hidráulica, redes de saneamiento, descriptor, indicador

\section{Introducción}

El desarrollo de proyectos como estrategia de aprendizaje es una metodología en la que los alumnos planean, implementan y evalúan trabajos completos que tienen aplicación en el mundo real más allá del aula (Blank, 1997; Dickinson, et al, 1998; Galeana, 2003); pero que a su vez, deben servir como trabajo académico para la evaluación particular de la asignatura en que se enmarcan.

Así pues, la evaluación de los estudiantes a través de los trabajos por ellos desarrollados es una tarea tremendamente compleja en ocasiones, y que conlleva muchas implicaciones para ellos. Asimismo, las tareas de evaluación en tiempos más recientes no sólo conllevan una toma de decisiones sobre cómo el alumno ha adquirido los conocimientos, sino que implican un análisis por parte del profesor de cómo se han implementado en sus desarrollos las diferentes competencias transversales que comienzan a formar parte de los programas educativos, particularmente de la Universitat Politècnica de València (UPV). El caso de asignaturas relacionadas con el área de conocimiento de Ingeniería Hidráulica no es diferente, y el docente tiene que alinear los conocimientos impartidos con los resultados de aprendizaje no solo de la materia en cuestión sino de aquellas competencias transversales (CT) que trabaje o sea punto de control. Dentro de las 13 CT que incluye el Plan Estratégico UPV2020 (PEUPV2020) del Vicerrectorado de Estudios, Calidad y Acreditación, se encuentra la CT-05 'Diseño y Proyecto' (UPV, 2015).

La evaluación es necesaria para regular el aprendizaje, conocer por parte del profesor y las estructuras que proponen el plan de estudios, que las asignaturas que se ofertan con los contenidos y competencias asignadas, son aprendidas por los alumnos. De otro lado, para informar a los propios alumnos y a la sociedad de la que forman parte, de los resultados de su aprendizaje, valorados bajo criterios comunes para todos, los cuales puedan ser cuantificados y comparados (Trujillo, 2013; Harwell, S., 1997). Es más, la evaluación diseñada como parte del proceso de aprender es más enriquecedora que como cuantificación de los resultados obtenidos, al menos en la experiencia de los autores, desde el punto de vista del docente.

Las estrategias de evaluación en la actualidad son variadas, sin embargo entre ellas, en este documento se analiza en profundidad aquella que evalúa los conocimientos en un tema determinado (en este caso las redes de drenaje y saneamiento en el marco curricular del Máster en Ingenieria Hidráulica y Medio Ambiente) a través del desarrollo de un trabajo, asimilado a un proyecto. Este tipo de aprendizaje es motivador para el alumno, porque le permite entre otras cosas, tener un producto final completo, lo que le da una visión más amplia de la asignatura que la evaluación centrada en otro tipo de estrategias con cuestionarios de menor alcance. 
Además, esta asignatura cuenta con una gran diversidad de orígenes de alumnos (estudiantes propios de la UPV, de otras Universidades españolas, de países del Norte de Europa e Iberoamérica ). En la mayoría de las Universidades de las cuales proceden los estudiantes no cuentan con un sistema de trabajo y evaluación de competencias transversales. Este hecho determina que tanto profesores como alumnos, tengan que realizar un esfuerzo en la programación y seguimiento de la asignatura para poder trabajar y evaluar un nivel de competencia de dominio III (Master), intentando alcanzar el máximo grado de aprendizaje en los diferentes descriptores.

\section{Objetivos}

La asignatura en que se implementa la presente metodología es 'Redes de saneamiento', enmarcada en el Master Universitario en Ingeniería Hidraulica y Medio Ambiente. Los alumnos que acceden a esta asignatura ya tienen un grado de conocimiento y madurez que permiten el desarrollo de proyectos o tareas complejas de manera muy adecuada. Tanto los conocimientos previos necesarios, como el desarrollo de otras competencias ,como el trabajo autónomo o el trabajo en equipo han sido suficientemente desarrollados por los alumnos, que ya se encuentran en esta fase final de su aprendizaje, perfectamente capacitados para proponer ideas de proyectos con mucha entidad.

Los proyectos desarrollados por los alumnos deben cumplir con ciertos condicionantes, que serán tenidos en cuenta a través de su evaluación posterior:

1) Deben estar centrados en los contenidos de la asignatura, pero reconociendo aspectos previos que ya han sido adquiridos en otras asignaturas relacionadas.

2) Deben estar claramente definidos, con un enunciado que permita plantear condiciones iniciales, un desarrollo y un final.

3) Deben proponer problemas interesantes y relacionados con la asignatura y el contexto curriucular del Máster en que se integran.

4) Deben permitir al alumno tener un producto final tangible que pueda compartir con sus compañeros.

5) Debe suponer una oportunidad para el alumno y sus compañeros de reflexionar sobre los temas tratados y de comparar diferentes soluciones.

En este sentido, el objetivo de implementar metodologías de evaluación por proyectos en una asignatura como la que se presenta en esta contribución, permite a los alumnos comprobar su grado de conocimiento y capacidad de comunicación en el tema que a su vez tiene una gran ambientalización curricular por las implicaciones que tienen las redes de saneamiento en la gestión de problemas medioambientales. Así, se persiguen objetivos que permiten implementar diferentes estrategias relacionadas con el proyecto de CT de la UPV, importante para la Institución como colectivo.

De la misma manera, los conocimientos y competencias adquiridos se vuelven a implementar con fuerza en fases finales del presente Máster. Al ser un momento final en el currículum de los estudiantes, el desarrollo de su Trabajo Final de Máster con un contenido muy relacionado con los temas que se implementan en la asignatura de Redes de Saneamiento, permite a algunos de los estudiantes, volver a aplicar lo que se ha desarrollado en esta asignatura, con mucha mayor complejidad en algunos de los trabajos ya defendidos en dicha titulación. 


\section{Desarrollo de la innovación}

El desarrollo de la competencia transversal 'Diseño y Proyecto' aplicada a la asignatura de Redes de Saneamiento tiene por objeto:

1) En un Nivel de Dominio III, ser capaz de trabajar la competencia y evaluarla, teniendo en cuenta la gran diversidad en el origen de alumnos que la cursan. Además, se debe considerar que en pocas ocasiones el alumno ha trabajado o ha sido evaluado por competencias en sus Universidades donde cursaron los estudios de Grado. Esto dota de una mayor dificultad a la hora de programar el nivel de Dominio III, puesto que los niveles I y II no han sido trabajados previamente por el alumno.

2) Planificar el desarrollo temporal del proyecto definido a través de las diferentes fases presenciales y no presenciales. En la fase presencial el alumno adquiere los resultados de aprendizaje necesarios que le permiten poner en práctica de manera progresiva el desarrollo del proyecto. Es el desarrollo del proyecto, el que se realiza de forma no presencial, con ayuda de los recursos disponibles a través de la plataforma PoliformaT. En la fase no presencial, el alumno trabaja el aprendizaje autónomo, el cual se ve complementado y/o apoyado con la tutorización del profesor a lo largo del tiempo que la actividad se encuentra en proceso de realización para lograr alcanzar con éxito los resultados de aprendizaje propuestos (Figura 1).

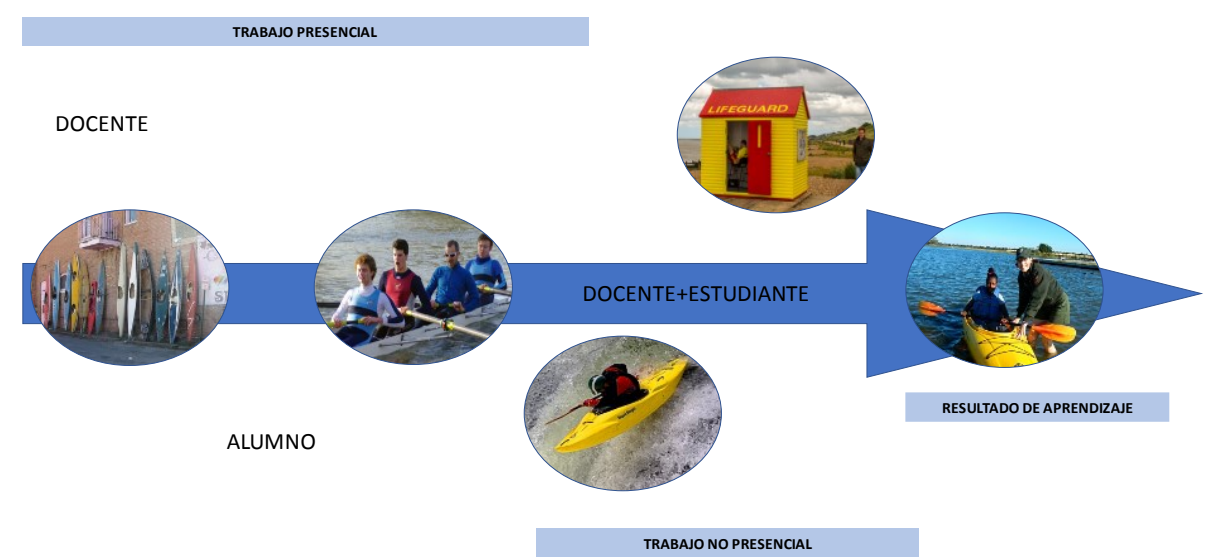

Fig 1. Evolución temporal del trabajo presencial y no presencial hasta alcanzar el resultado de aprendizaje

3) Desarrollar una transición de la nota numérica tradicionalmente establecida en el trabajo a establecer un grado de adquisición de la competencia mediante indicadores y descriptores, a través de una rúbrica que contemple los resultados de aprendizaje a alcanzar por el alumno en dicho proyecto. No obstante, esta transición tiene por finalidad que una vez establecida la rúbrica, pueda operarse de forma inversa. Conocido el grado de adquisición de cada uno de los indicadores

(cc) EY-NC-ND 2017, Universitat Politècnica de València 
definidos, se puede establecer un valor numérico que pueda ser tenido en cuenta para la nota del expediente del alumno, puesto que el trabajo representa un acto de evaluación dentro de la Guía Docente de la asignatura con un peso del 10\% sobre la calificación final.

\section{Resultados}

\subsection{Resultados del seguimiento del alumno}

La asignatura "Redes de saneamiento" se fundamenta en dos asignaturas obligatorias del plan de estudios del Máster en Ingeniería Hidráulica y Medio Ambiente del semestre A del primer curso: "Hidráulica avanzada" e "Hidrología superficial y subterránea".

La asignatura "Hidráulica Avanzada" es una asignatura troncal que tiene como objetivo lograr que todos los alumnos del Máster tengan unos conocimientos de hidráulica avanzada suficientes para poder desarrollar proyectos relacionados con la ingeniería hidráulica. Sirve de base para poder seguir profundizando, en el segundo semestre, en las asignaturas de intensificación relacionadas. En concreto, establece las bases para el cálculo hidráulico en lámina libre de colectores que se desarrollan en la asignatura "Redes de saneamiento" y que, en particular, son objeto de la práctica que aquí se analiza.

La asignatura "Hidrología superficial y subterránea" es igualmente una asignatura troncal del primer semestre en la que se establecen, en relación con "Redes de saneamiento" los conocimientos teóricos a problemas reales relacionados con la estimación del riesgo de las crecidas, profundizando en los métodos necesarios para el análisis de la precipitación, la producción de escorrentía y su propagación en la cuenca.

Así pues, la existencia previa en el plan de estudios de estas dos asignaturas garantiza que el alumno que aborda la asignatura "Redes de saneamiento" ha cursado, independientemente de su procedencia, los conceptos necesarios para poder abordar con solvencia esta asignatura de intensificación. Por otra parte, el alumno que se enfrenta a esta asignatura es un alumno de máster y, por tanto, con un nivel de madurez que le permite enfrentarse a una actividad en la cual pretenda demostrar que es capaz de alcanzar el nivel III de dominio de la competencia transversal CT-05, aunque no haya trabajado específicamente previamente los niveles I y II (propios de titulaciones de grado).

No obstante, en las mencionadas asignaturas previas del primer semestre, el alumno ha sido evaluado, entre otros, mediante el análisis de casos, planteando el análisis y la resolución de una determinada situación que presenta problemas de solución múltiple, a través de la reflexión y el diálogo para un aprendizaje grupal, integrado y significativo. Esto supone sin duda, un buen entrenamiento previo a la resolución completa de un proyecto, en unas condiciones más ambiciosas y donde el contexto previo está, deliberadamente, poco estructurado por parte del profesor. En cualquier caso, el apoyo por parte del profesor, tanto en la fase presencial como en la no presencial a través de las tutorías, es una pieza fundamental para conseguir que el alumno consiga demostrar un nivel de dominio III en esta competencial transversal, la cual debe adquirir sin haber trabajado previamente niveles anteriores. 


\subsection{Resultados de planificación temporal del trabajo}

El proyecto se comienza a desarrollar en una fase presencial de dos horas de duración en la cuarta semana del curso y en una fase no presencial de duración un mes, hasta la octava semana del curso, momento en el cual, el alumno debe entregar la memoria de la actividad.

La fase presencial ocurre cuando, en las tres primeras semanas del curso, el alumno ha adquirido los conocimientos referentes a las generalidades del diseño de una red de colectores y a los métodos hidrológicos e hidráulicos de diseño de este tipo de redes. Durante las dos horas de esta fase presencial, el profesor dirige a los alumnos a reflexionar y trabajar los puntos clave para la realización de la actividad, desarrollando en cada paso una puesta en común que subraye estos aspectos fundamentales.

En primer lugar, se realiza una lectura conjunta con los alumnos del enunciado de la práctica, discutiendo en grupo la problemática planteada y las líneas maestras conceptuales de la solución. A continuación, cada alumno desarrolla una propuesta de topología de la red, enfrentándose a la toma de ciertas decisiones que condicionarán en todo el desarrollo posterior del proyecto. Conforme surgen problemas y dudas por parte de los alumnos, el profesor las resuelve y remarca las estrategias más adecuadas para establecer dicha topología. Establecida la topología de la red, se analiza en grupo la información de partida consistente en la ortofotografía de la zona y cómo a partir de ésta deben asociarse los usos del suelo a los parámetros del modelo de cálculo hidrológico. A continuación, el profesor plantea a los alumnos la idea general para proceder a la delimitación y asignación de cuencas tributarias y deja que los alumnos se enfrenten a este paso del proceso de diseño. Por último, se trabajan los condicionantes para el encaje de los perfiles longitudinales de los distintos ramales de la red de colectores. Con esta actividad, concluye la sesión presencial de dos horas y a partir de este punto, comienza la fase no presencial de trabajo autónomo e individual del alumno.

La fase no presencial se extiende durante las siguientes cuatro semanas. Se estima en unas 10-15 horas el tiempo necesario para que el alumno complete el diseño de la red y lo plasme en la memoria requerida. Durante este mes, el profesor apoya con tutorías a los alumnos que así lo demandan para resolver los aspectos puntuales que hayan podido ir surgiendo durante la elaboración del proyecto.

\subsection{Propuesta de rúbrica}

El proyecto está estratificado en 9 ítems los cuales han servido tradicionalmente para establecer un valor numérico que computase en la nota final del alumno. Los autores de la presente comunicación han desarrollado una rúbrica (adaptada de la realizada por un equipo de profesores de la UPV y el ICE), la cual toma como referencia los indicadores propuestos a estos ítems, con el objetivo de no realizar una valoración numérica sino una valoración objetiva del grado alcanzado de los resultados de aprendizaje del alumno. Los ítems que tradicionalmente han sido corregidos son los que se adjuntan a continuación:

- Ítem 1 Establecimiento de la topología de la red

- Ítem 2 Justificación de los coeficientes de escorrentía adoptados

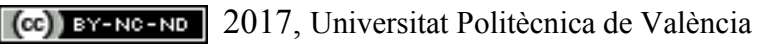


- Ítem 3 Delimitación y asignación de subcuencas

- Ítem 4 Encaje de los perfiles longitudinales de los colectores

- Ítem 5 Cálculo de los diámetros de los conductos

- Ítem 6 Cálculo de las velocidades en los conductos

- Ítem 7 Representación gráfica de los perfiles longitudinales

- Ítem 8 Expresión escrita en la memoria

- Ítem 9 Edición de la memoria

Todos los ítems tenían una puntuación máxima de un punto, a excepción del Ítem 5 cuya puntuación es de dos puntos. La suma total era de 10 puntos, repercutiendo un $10 \%$ sobre la nota final.

A continuación, en las Tablas 1 y 2, se muestran los indicadores evaluados en el desarrollo de la competencia transversal.

Tabla 1. Rúbrica de evaluación de las actividades para Nivel de dominio III

\begin{tabular}{|c|c|c|c|c|c|}
\hline \multirow{2}{*}{ INDICADOR } & \multicolumn{4}{|c|}{ DESCRIPTORES } & \multirow{2}{*}{\begin{tabular}{|} 
EVIDENCIAS DE \\
CADA UNO DE LO \\
INDICADORES
\end{tabular}} \\
\hline & $\begin{array}{l}\text { No Alcanzado } \\
\text { (D) }\end{array}$ & $\begin{array}{c}\text { En desarrollo } \\
\text { (C) }\end{array}$ & $\begin{array}{c}\text { Bien /adecuado } \\
\text { (B) }\end{array}$ & $\begin{array}{l}\text { Excelente } \\
\text { (A) }\end{array}$ & \\
\hline $\begin{array}{l}\text { 1. Valora las } \\
\text { necesidades en } \\
\text { un contexto } \\
\text { real de } \\
\text { intervención }\end{array}$ & $\begin{array}{c}\text { No las valora } \\
\text { o lo hace } \\
\text { incorrectame } \\
\text { nte }\end{array}$ & $\begin{array}{c}\text { Hace una } \\
\text { valoración en la } \\
\text { que comete } \\
\text { algún/os fallos/s o } \\
\text { en algunos casos } \\
\text { incorrecta }\end{array}$ & $\begin{array}{l}\text { Hace una } \\
\text { valoración } \\
\text { suficiente, } \\
\text { aunque no } \\
\text { completa, pero } \\
\text { siempre } \\
\text { correcta }\end{array}$ & $\begin{array}{l}\text { Hace una valoración } \\
\text { suficiente, correcta y } \\
\text { completa }\end{array}$ & \\
\hline $\begin{array}{c}\text { Evaluación } \\
\text { atendiendo a la } \\
\text { actividad }\end{array}$ & $\begin{array}{c}\text { El alumno no define } \\
\text { la necesidad de la } \\
\text { actuación en la } \\
\text { memoria }\end{array}$ & $\begin{array}{c}\text { El alumno cita la } \\
\text { necesidad de la } \\
\text { actuacion pero no } \\
\text { la justifica }\end{array}$ & $\begin{array}{c}\text { El alumno define la } \\
\text { necesidad y } \\
\text { desarrolla una } \\
\text { justificación de la } \\
\text { misma en la memoria } \\
\text { con justificaciones } \\
\text { correctas pero } \\
\text { incompletas }\end{array}$ & $\begin{array}{c}\text { El alumno enumera y } \\
\text { justifica la necesidad de } \\
\text { actuación de forma } \\
\text { correcta y completa }\end{array}$ & $\begin{array}{l}\text { Existe el } \\
\text { apartado en } \\
\text { la memoria } \\
\text { "Necesidad } \\
\text { de la } \\
\text { actuación" }\end{array}$ \\
\hline $\begin{array}{l}\text { 2. Concreta } \\
\text { objetivos } \\
\text { operativos } \\
\text { del proyecto } \\
\text { yestablece } \\
\text { plazos }\end{array}$ & $\begin{array}{c}\text { No concreta } \\
\text { objetivos ni plazos }\end{array}$ & $\begin{array}{l}\text { Concreta } \\
\text { algunos } \\
\text { objetivos y } \\
\text { algunos plazos }\end{array}$ & $\begin{array}{c}\text { Concreta } \\
\text { objetivos } \\
\text { operativos del } \\
\text { proyecto y } \\
\text { establece plazos }\end{array}$ & $\begin{array}{l}\text { Concreta todos } \\
\text { los objetivos y } \\
\text { todos los plazos } \\
\text { con perspectivas } \\
\text { de futuro }\end{array}$ & \\
\hline $\begin{array}{c}\text { Evaluación } \\
\text { atendiendo a la } \\
\text { actividad }\end{array}$ & $\begin{array}{c}\text { El alumno no define } \\
\text { en la memoria } \\
\text { entregable los } \\
\text { objetivos del } \\
\text { proyecto }\end{array}$ & $\begin{array}{l}\text { El alumno no } \\
\text { define todos los } \\
\text { objetivos }\end{array}$ & $\begin{array}{c}\text { El alumno define } \\
\text { todos los objetivos } \\
\text { del proyecto }\end{array}$ & $\begin{array}{c}\text { El alumno define todos } \\
\text { los objetivos del } \\
\text { proyecto, estableciendo } \\
\text { una priorización de los } \\
\text { mismos y expresándolos }\end{array}$ & $\begin{array}{c}\text { La } \\
\text { memoria } \\
\text { contiene el } \\
\text { apartado de } \\
\text { objetivos }\end{array}$ \\
\hline $\begin{array}{c}\text { 3. Planifica } \\
\text { las acciones } \\
\text { y prevé } \\
\text { contingencia } \\
\text { s }\end{array}$ & $\begin{array}{l}\text { No planifica las } \\
\text { acciones ni } \\
\text { prevé } \\
\text { contingencias }\end{array}$ & $\begin{array}{c}\text { Planifica las } \\
\text { acciones y/o } \\
\text { contingencias, } \\
\text { pero comete } \\
\text { fallos: no lo } \\
\text { hace de la } \\
\text { manera más } \\
\text { adecuada }\end{array}$ & $\begin{array}{l}\text { Planifica las } \\
\text { acciones y } \\
\text { prevé las } \\
\text { contingencias }\end{array}$ & $\begin{array}{c}\text { Planifica las } \\
\text { acciones y además } \\
\text { prevé las } \\
\text { contingencias } \\
\text { proponiendo } \\
\text { alternativas }\end{array}$ & \\
\hline
\end{tabular}




\begin{tabular}{|c|c|c|c|c|c|}
\hline $\begin{array}{c}\text { Evaluación } \\
\text { atendiendo a la } \\
\text { actividad }\end{array}$ & \begin{tabular}{|c|} 
El alumno no planifica \\
el proceso de resolución \\
del proyecto, no \\
dibujando los perfiles \\
longitudinales finales.
\end{tabular} & $\begin{array}{c}\text { El alumno planifica la } \\
\text { metodología de } \\
\text { resolución pero no es } \\
\text { correcta, en ningún } \\
\text { caso. }\end{array}$ & $\begin{array}{l}\text { El alumno planifica la } \\
\text { metodología de } \\
\text { resolución de forma } \\
\text { correcta, pero no es } \\
\text { capaz de desarrollar } \\
\text { todos los perfiles }\end{array}$ & $\begin{array}{c}\text { El alumno planifica la } \\
\text { metodología de resolución } \\
\text { de forma correcta y justifica } \\
\text { la toma de decisiones, } \\
\text { demostrandolo con los } \\
\text { perfiles finales longitudiales } \\
\text { de energía }\end{array}$ & $\begin{array}{l}\text { El alumno además de } \\
\text { definirlo en la } \\
\text { memoria, finaliza el } \\
\text { proyecto con la } \\
\text { representación de los } \\
\text { perfiles longitudinales }\end{array}$ \\
\hline
\end{tabular}

Tabla 2. Rúbrica de evaluación de las actividades para Nivel de dominio III (Continuación)

\begin{tabular}{|c|c|c|c|c|c|}
\hline \multirow{2}{*}{ INDICADOR } & \multicolumn{4}{|c|}{ DESCRIPTORES } & \multirow{2}{*}{$\begin{array}{l}\text { EVIDENCIAS DE } \\
\text { CADA UNO DE LOS } \\
\text { INDICADORES }\end{array}$} \\
\hline & $\begin{array}{l}\text { No Alcanzado } \\
\text { (D) }\end{array}$ & $\begin{array}{c}\text { En desarrollo } \\
\text { (C) }\end{array}$ & $\begin{array}{c}\text { Bien /adecuado } \\
\text { (B) }\end{array}$ & $\begin{array}{l}\text { Excelente } \\
\text { (A) }\end{array}$ & \\
\hline $\begin{array}{c}\text { 4. Gestiona } \\
\text { adecuadamente } \\
\text { los recursos } \\
\text { disponibles }\end{array}$ & $\begin{array}{l}\text { No gestiona los } \\
\text { recursos } \\
\text { disponibles }\end{array}$ & $\begin{array}{l}\text { Gestiona los } \\
\text { recursos pero } \\
\text { comete fallos: } \\
\text { no lo hace de la } \\
\text { manera más } \\
\text { adecuada }\end{array}$ & $\begin{array}{l}\text { Gestiona } \\
\text { adecuada } \\
\text { mente los } \\
\text { recursos } \\
\text { disponibl } \\
\text { es } \\
--.-\end{array}$ & $\begin{array}{l}\text { Gestiona } \\
\text { adecuadamente } \\
\text { todos los } \\
\text { recursos } \\
\text { disponibles }\end{array}$ & \\
\hline $\begin{array}{c}\text { Evaluación } \\
\text { atendiendo a la } \\
\text { actividad }\end{array}$ & $\begin{array}{c}\text { El alumno no es capaz } \\
\text { de analizar la } \\
\text { informaicón disponible } \\
\text { en PoliformaT para } \\
\text { desarrollar el proyecto }\end{array}$ & $\mid \begin{array}{c}\text { El alumno es capaz de } \\
\text { analizar la } \\
\text { información, la } \\
\text { enumera en la } \\
\text { memoria pero no la } \\
\text { utiliza correctamente }\end{array}$ & $\begin{array}{l}\text { El alumno es capaz de } \\
\text { analizar la informción, } \\
\text { enumerandola en la } \\
\text { memoria y utilizándola } \\
\text { de una forma correcta }\end{array}$ & \begin{tabular}{|} 
El alumno utiliza toda la \\
información disponible, la \\
enumera en la memoria y la \\
complementa con otros \\
recursos buscados por él \\
mismo, mejorando la \\
informaicón de partida del \\
proyecto (modelos digitales, \\
catálogos de fabricantes, \\
información urbanistica...)
\end{tabular} & $\begin{array}{c}\text { El alumno procesa } \\
\text { la información } \\
\text { disponible para } \\
\text { desarrollar el } \\
\text { proyecto, } \\
\text { presentándola en } \\
\text { la memoria }\end{array}$ \\
\hline $\begin{array}{c}\text { 5. Realiza un } \\
\text { seguimiento } \\
\text { de la } \\
\text { implementac } \\
\text { ión del } \\
\text { proyecto }\end{array}$ & $\begin{array}{l}\text { No evidencia el } \\
\text { seguimiento }\end{array}$ & $\begin{array}{l}\text { Hay algunas } \\
\text { evidencias de } \\
\text { realizar el } \\
\text { seguimiento }\end{array}$ & $\begin{array}{c}\text { Hace un } \\
\text { seguimiento } \\
\text { coherente del } \\
\text { proyecto hacia } \\
\text { la consecución } \\
\text { de los objetivos, } \\
\text { con alguna } \\
\text { omisión }\end{array}$ & $\begin{array}{l}\text { Hace un seguimiento } \\
\text { completo y } \\
\text { coherente del } \\
\text { proyecto hacia la } \\
\text { consecución de los } \\
\text { objetivos }\end{array}$ & \\
\hline $\begin{array}{c}\text { Evaluación } \\
\text { atendiendo a la } \\
\text { actividad }\end{array}$ & $\begin{array}{c}\text { El alumno únicamente } \\
\text { es capaz de definir la } \\
\text { topología de la red }\end{array}$ & $\begin{array}{c}\text { El alumno define la } \\
\text { topología de la red, es } \\
\text { capaz de establecer de } \\
\text { forma correcta las } \\
\text { subcuencas de la red } \\
\text { (Anexo } 2 \text { y } 3 \text { ), pero } \\
\text { desarrolla de forma } \\
\text { incorrecta la } \\
\text { determinación de los } \\
\text { diametros (Anexo 4) }\end{array}$ & $\begin{array}{c}\text { El alumno define } \\
\text { correctamente las } \\
\text { subcuencas y perfiles, } \\
\text { determinando de forma } \\
\text { correcta los diámetros } \\
\text { (Anexo 4) y comprueba } \\
\text { las velocidades con } \\
\text { diámetros comerciales } \\
\text { (Anexo 5) }\end{array}$ & $\begin{array}{c}\text { El alumno desarrolla de } \\
\text { forma correcta todos los } \\
\text { anexos, desarolla los planos } \\
\text { de la red solicitada tanto en } \\
\text { planta como en perfil, } \\
\text { justificando de forma } \\
\text { escrita toda la toma de } \\
\text { decisiones }\end{array}$ & $\begin{array}{l}\text { El alumno desarrolla } \\
\text { los } 7 \text { puntos } \\
\text { considerados en el } \\
\text { enunciado del } \\
\text { proyecto dentro de la } \\
\text { memoria, } \\
\text { justificándolo en los } \\
\text { diferentes anexos }\end{array}$ \\
\hline $\begin{array}{c}\text { 6. Gestiona } \\
\text { adecuadamente } \\
\text { los riesgos del } \\
\text { proyecto }\end{array}$ & $\begin{array}{l}\text { No evidencia la } \\
\text { gestión de los } \\
\text { riesgos }\end{array}$ & $\begin{array}{l}\text { Gestiona los } \\
\text { riesgos pero } \\
\text { comete fallos: } \\
\text { no lo hace de } \\
\text { la manera más } \\
\text { adecuada }\end{array}$ & $\begin{array}{l}\text { Gestiona } \\
\text { adecuada } \\
\text { mente los } \\
\text { riesgos } \\
\text { del } \\
\text { proyecto }\end{array}$ & $\begin{array}{l}\text { Gestiona } \\
\text { adecuadamente } \\
\text { todos los riesgos } \\
\text { del proyecto }\end{array}$ & \\
\hline
\end{tabular}

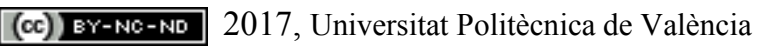




\begin{tabular}{|c|c|c|c|c|c|}
\hline $\begin{array}{c}\text { Evaluación } \\
\text { atendiendo a la } \\
\text { actividad }\end{array}$ & {$\left[\begin{array}{c}\text { El alumno no toma los } \\
\text { coeficientes necesarios } \\
\text { para determinar la } \\
\text { producción de } \\
\text { escorrentia asi como los } \\
\text { coeficientes de } \\
\text { rugosidad de la } \\
\text { conducciones. No } \\
\text { respeta la normativa } \\
\text { vigente }\end{array}\right.$} & $\mid \begin{array}{c}\text { El alumno toma los } \\
\text { coeficientes de } \\
\text { escorrentía y } \\
\text { rugosidad de manera } \\
\text { correcta, pero los } \\
\text { considera inferiores a } \\
\text { los valores } \\
\text { recomendados en la } \\
\text { bibliografia, teniendo } \\
\text { en cuenta la normativa } \\
\text { vigente }\end{array}$ & $\begin{array}{c}\text { El alumno toma los } \\
\text { coeficientes de forma } \\
\text { correcta, tomando } \\
\text { valores usualmente } \\
\text { utilizados por la } \\
\text { bibliografia, teniendo en } \\
\text { cuenta la normativa } \\
\text { vigente }\end{array}$ & $\begin{array}{c}\text { El alumno toma valores de } \\
\text { coeficientes correctos, y } \\
\text { desarrolla un ánalisis de } \\
\text { sensibilidad, discutiendo la } \\
\text { diferencia de los resultados, } \\
\text { teniendo en cuenta la } \\
\text { normativa vigente }\end{array}$ & $\begin{array}{l}\text { Justificación de los } \\
\text { esquemas de } \\
\text { funcionamiento } \\
\text { seleccionados y } \\
\text { adaptación a la } \\
\text { normativa }\end{array}$ \\
\hline
\end{tabular}

La tabla 3 muestra la correlación entre los 9 ítems y los 6 indicadores definidos, así como el peso ponderado del indicador para a partir del grado alcanzado en la competencia, dotar de un valor numérico para poder ser tenido en cuenta en el expediente del alumno en la evaluación de las competencias específicas.

Tabla 3. Correlación entre los indicadores e items

\begin{tabular}{|cl|c|c|}
\hline \multicolumn{2}{|c|}{ Indicador } & Ítem & Peso ponderado \\
& & & del indicador \\
\hline $1 . \quad$ Valora necesidades & Ítem 9 & 0.1 \\
\hline $2 . \quad$ Concreta objetivos & Ítem 8 & 0.1 \\
\hline $3 . \quad$ Planifica acciones & Ítem 7 & 0.1 \\
\hline $4 . \quad$ Gestiona recursos & Ítems 1 y 4 & 0.15 \\
\hline $5 . \quad$ Realiza seguimiento de implementación & Ítems 3 y 5 & 0.35 \\
\hline $6 . \quad$ Gestiona los riesgos & Ítems 2 y 6 & 0.20 \\
\hline
\end{tabular}

En cuanto a los criterios para definir la valoración de la competencia considerando los 6 indicadores se adjuntan a continuación la Tabla 4 que muestra dicha equivalencia propuesta.

Tabla 4. Correlaciones principales entre grado alcanzado de la competencia e indicadores

\begin{tabular}{|l|c|}
\hline \multicolumn{1}{|c|}{ Grado alcanzado en los indicadores } & Calificación \\
\hline $\begin{array}{l}\text { Todos los indicadores son A } \\
\text { Existen hasta dos indicadores calificados como B que no coinciden con los indicadores de 4 a 6, } \\
\text { siendo éstos A }\end{array}$ & A \\
\hline $\begin{array}{l}\text { Dos indicadores de 4 a 6 son B, y al menos uno de 1 a 3 es B } \\
\text { Los tres indicadores de 4 a 6 son B, y cómo máximo solo existe un indicador C de 1 a 3 }\end{array}$ & B \\
\hline $\begin{array}{l}\text { Uno de los indicadores de 4 a 6 es C, dando igual el número de A existentes } \\
\text { Todos los indicadores de 4 a 6 son B, y existen dos indicadores C o uno D }\end{array}$ \\
\hline $\begin{array}{l}\text { Existe un indicador de 4 a 6 evaluado como D } \\
\text { Todos los indicadores entre 4 y 6 son inferiores a A, y al menos existen dos indicadores de 1 a 3 }\end{array}$ \\
\hline
\end{tabular}

(c)) EY-NG-ND 2017, Universitat Politècnica de València 


\subsection{Resultados obtenidos}

Los resultados obtenidos en el cuatrimestre B del curso 2015/2016, en el cual estuvieron matriculados 23 alumnos, fueron los que se adjuntan a continuación en la Tabla 5. En esta tabla pueden observarse los resultados de las calificaciones y correlaciones para cada uno de los alumnos, presentados de forma anónima. 
Tabla 5. Correlación entre los indicadores e items

\begin{tabular}{|c|c|c|c|c|c|c|c|}
\hline \multirow{2}{*}{ ALUMNO } & \multicolumn{6}{|c|}{ INDICADORES } & \multirow{2}{*}{$\begin{array}{l}\text { CALIFICACIÓN } \\
\text { GLOBAL }\end{array}$} \\
\hline & 1 & 2 & 3 & 4 & 5 & 6 & \\
\hline A01 & $\mathrm{A}$ & A & A & A & A & A & A \\
\hline $\mathrm{A} 02$ & $\mathrm{~A}$ & $\mathrm{~A}$ & B & A & B & A & A \\
\hline $\mathrm{A} 03$ & A & C & C & B & B & B & B \\
\hline A04 & D & C & D & B & C & C & C \\
\hline A05 & A & A & C & A & B & A & B \\
\hline A06 & B & $\mathrm{A}$ & A & $\mathrm{A}$ & B & $\mathrm{A}$ & A \\
\hline A07 & $\mathrm{A}$ & $\mathrm{A}$ & C & B & $\mathrm{A}$ & C & B \\
\hline A08 & B & A & C & A & A & B & B \\
\hline A09 & D & C & C & A & A & B & C \\
\hline A10 & $\mathrm{A}$ & $\mathrm{A}$ & B & B & B & B & B \\
\hline A11 & A & A & C & A & B & A & B \\
\hline A12 & $\mathrm{B}$ & $\mathrm{B}$ & D & A & $\mathrm{B}$ & $\mathrm{A}$ & B \\
\hline A13 & A & $\mathrm{A}$ & D & $\mathrm{A}$ & B & B & C \\
\hline A14 & B & C & D & $D$ & $\mathrm{D}$ & C & D \\
\hline A15 & B & A & A & A & C & A & B \\
\hline A16 & D & A & B & A & C & B & B \\
\hline A17 & A & A & A & A & B & A & A \\
\hline A18 & A & $\mathrm{A}$ & $D$ & $\mathrm{~A}$ & C & B & B \\
\hline A19 & $\mathrm{A}$ & $\mathrm{A}$ & A & $\mathrm{A}$ & B & B & B \\
\hline $\mathrm{A} 20$ & D & B & B & A & C & A & B \\
\hline A21 & D & A & B & A & B & B & B \\
\hline A22 & D & D & D & A & C & C & D \\
\hline A23 & A & A & B & A & C & A & B \\
\hline
\end{tabular}

Finalmente, en la Tabla 6 se comparan las notas numéricas evaluadas mediante ítems $\left(\mathrm{N}_{\mathrm{i}}\right)$, tal y como se ha venido desarrollando, y las notas numéricas obtenidas por competencias cuando se hace la transformación numérica $\left(\mathrm{N}_{\mathrm{c}}\right)$. Para desarrollar esta equivalencia se tienen en cuenta, los pesos ponderados de la Tabla 3, y se dota al descriptor de un valor igual a 5 cuando es $\mathrm{A} ; 4$ cuando es $\mathrm{B} ; 2$ cuando es $\mathrm{C}$; y 0.5 cuando es D.

La tabla describe que las calificaciones obtenidas son muy similares no existiendo cambios sustanciales en las notas obtenidas, manteniéndose los alumnos que obtienen una muy buena calificación por ítems, así como aquellos que no han conseguido superar la asignatura porque no han logrado alcanzar los resultados de aprendizaje. 
Tabla 6. Comparación de notas obtenidas mediante items y por equivalencia de competencias

\begin{tabular}{|c|c|c|c|c|c|c|c|}
\hline ALUMNO & $\left(\mathbf{N}_{\mathrm{i}}\right)$ & $\left(\mathbf{N}_{c}\right)$ & {$\left[\mathbf{N}_{\mathrm{c}}-\mathbf{N}_{\mathrm{i}}\right]$} & ALUMNO & $\left(\mathbf{N}_{\mathrm{i}}\right)$ & $\left(\mathbf{N}_{\mathbf{c}}\right)$ & {$\left[\mathbf{N}_{\mathrm{c}}-\mathbf{N}_{\mathrm{i}}\right]$} \\
\hline A01 & 10 & 10 & 0 & A13 & 8 & 8 & 0 \\
\hline A02 & 9.5 & 9.1 & -0.4 & A14 & 1.5 & 2.6 & 1.1 \\
\hline A03 & 7 & 7.4 & 0.4 & A15 & 8.5 & 7.7 & -0.8 \\
\hline A04 & 4 & 4 & 0 & A16 & 7 & 6.4 & -0.6 \\
\hline A05 & 9 & 8.7 & -0.3 & A17 & 9.5 & 9.3 & -0.2 \\
\hline A06 & 8.5 & 9.1 & 0.6 & A18 & 7.25 & 6.6 & -0.65 \\
\hline A07 & 7.5 & 7.9 & 0.4 & A19 & 8.5 & 8.9 & 0.4 \\
\hline A08 & 8.5 & 8.8 & 0.3 & A20 & 6.5 & 6.6 & 0.1 \\
\hline A09 & 7 & 7.5 & 0.5 & A21 & 7.5 & 7.8 & 0.3 \\
\hline A10 & 8.25 & 8.4 & 0.15 & A22 & 3.5 & 4 & 0.5 \\
\hline A11 & 9 & 8.7 & -0.3 & A23 & 7.75 & 7.7 & -0.05 \\
\hline A12 & 8 & 8 & 0 & & & & \\
\hline
\end{tabular}

\section{Conclusiones}

En la presente comunicación se ha mostrado un caso de estudio de nivel de Dominio III, concretamente de la asignatura Redes de Saneamiento, la cual ha permitido comprobar su grado de conocimiento y capacidad de comunicación, en un tema que a su vez tiene una gran ambientalización curricular por las implicaciones que tienen las redes de saneamiento en la gestión de problemas medioambientales.

El desarrollo se ha establecido a traves de la descripción de una metodología de trabajo la cual ha combinado la tarea presencial y no presencial de los alumnos. Evaluada la competencia transversal Diseño y Proyecto, un $78.26 \%$ de los alumnos han adquirido con un un nivel adecuado o excelente dicha competencia, mostrando el éxito de la misma.

Para evaluar dicha competencia, los autores han propuesto una rúbrica la cual han adaptado a partir de los indicadores propuestos por el Instituto de Ciencias de la Educación de la UPV. Estos indicadores han sido descritos en función de los items que tradicionalmente han sido evaluados en dicho proyecto.

Finalmente, se ha mostrado la posibilidad de trabajar de un modo inverso a lo realizado hasta la fecha. A partir de los grados alcanzados en cada indicador, éstos han sido ponderados y se ha obtenido una nota númerica, la cual es introducida en el expediente académico para valorar las competencias generales y específicas de la asignatura. Tras realizar la transformación se ha observado que las notas obtenidas son muy similares, no mostrando ni variando los resultados finales del expediente académico del alumno.

(c)) EY-NC-ND 2017, Universitat Politècnica de València 


\section{Referencias}

TrujILlo SÁEZ, F. (2013) Aprendizaje basado en proyectos. Formación en Red. Instituto Nacional de Tecnologías Educativas y de Formación del Profesorado .

Blank, W. (1997). Authentic instruction. In W.E. Blank \& S. Harwell (Eds.), Promising practices for connecting high school to the real world (pp. 15-21). Tampa, FL: University of South Florida. (ERIC Document Reproduction Service No. ED407586)

Dickinson, K.P., Soukamneuth, S., Yu, H.C., Kimball, M., D’Amico, R., PrRy, R., et al. (1998). Providing educational services in the Summer Youth Employment and Training Program [Technical assistance guide]. Washington, DC: U.S. Department of Labor, Office of Policy \& Research. (ERIC Document Reproduction Service No. ED420756)

Galeana DE LA O. L. (2003) Aprendizaje basado en proyectos. Universidad de Colima. Mexico.

Harwell, S. (1997). Project-based learning. In: W. E. Blank and S. Harwell (Eds.), Promising practices for connecting high school to the real world (pp. 23-28). Tampa, FL: University of South Florida. (ERIC Document Reproduction Service No. ED 407586).

UPV (2015) "Plan estratégico 2015-2020". Universidad Politécnica de Valencia. [Consultado 20 marzo de 2017] 